\title{
Cirugía cardiovascular libre de opioides: un estudio prospectivo observacional
}

\author{
Cukierman D. ${ }^{1}$, Fernández E. ${ }^{1}$ \\ 1 CEMIC, CABA, Argentina.
}

Introducción: El objetivo de este estudio fue comparar la anestesia general libre de opioides con la anestesia general balanceada con remifentanilo en cirugía cardiovascular (CCV) en términos de dolor agudo postoperatorio. Si bien en la práctica habitual en CCV se suelen utilizar grandes dosis de opiáceos la tendencia actual consiste en minimizar las dosis de los mismos. Presentamos como alternativa la realización de una anestesia totalmente libre de opioides para CCV.

Métodos: Se presentan resultados parciales de un estudio prospectivo observacional que está siendo realizado en el Hospital Universitario CEMIC. La hipótesis del mismo consiste en que la anestesia libre de opioides (OFA por sus siglas en inglés) permite un mejor control del dolor medido por escala verbal numérica y menores requerimientos de morfina en el postoperatorio que la anestesia basada en opiáceos en CCV. Se incluyeron todos los pacientes mayores de edad que requieran CCV programada en nuestra institución. Se excluyeron del análisis pacientes con antecedentes de dolor crónico, trastornos psiquiátricos medicados con drogas que puedan interferir con la correcta evaluación del dolor.

Comparamos la intensidad de dolor postoperatorio en pacientes que recibieron una anestesia general libre de opioides (grupo OFA) $(\mathrm{n}=8)$ contra aquellos que recibieron una anestesia general balanceada con remifentanilo $(\mathrm{n}=5)$. Durante la cirugía ambos grupos recibieron Sevoflorane $0,8 \%$ espirado, infiltración de herida y drenajes con ropivacaína $0,75 \%$ y ketorolac $1 \mathrm{mg} / \mathrm{kg}$. Como diferencias el grupo OFA recibió una infusión de ketamina $0,25-0,5 \mathrm{mg} / \mathrm{kg} / \mathrm{h}$ Lidocaina $1,5-3 \mathrm{mg} / \mathrm{kg} / \mathrm{h}$, Dexmedetomidina $0,3 \mathrm{mcg} / \mathrm{kg} / \mathrm{h}$ y esmolol $5 \mathrm{mcg} / \mathrm{kg} / \mathrm{min}$, paracetamol $10 \mathrm{mg} / \mathrm{kg}$, dipirona $20 \mathrm{mg} / \mathrm{kg}$; el grupo control recibió una infusión de remifentanilo $0,2-0,4 \mathrm{mcg} / \mathrm{kg} / \mathrm{h}$ y morfina $0,1 \mathrm{mg} / \mathrm{kg}$. Se realizaron mediciones de dolor según Escala Numérica Verbal (ENV) a las horas 0, 2, 4, 6, 12, 18, 24, 30, 36, 42 y 48 h respecto del momento de la extubación, además se midió consumo de morfina o equivalente durante las primeras $48 \mathrm{~h}$ postoperatorias, estadía hospitalaria y complicaciones postoperatorias.

Resultados: El dolor agudo postoperatorio fue de menor intensidad en el grupo OFA comparado con el grupo control (Grafico 1). El consumo promedio de opioides fue de 6,125 mg en el grupo OFA y 4,4 mg en el grupo control. No se observaron diferencias significativas en la estadía hospitalaria ni en las complicaciones postoperatorias salvo una tendencia a mayor duración de ventilación mecánica y mayor incidencia de excitacion psicomotriz y trastornos cognitivos en el grupo con opioides.

Conclusión: Creemos que la anestesia libre de opioides es una alternativa valida y segura en cirugía cardiovascular.

https://doi.org/10.25237/congresoclasa2019.87 